\title{
The challenges and progress in the management of reproduction in yaks
}

\author{
S J Yu \\ Faculty of Veterinary Medicine, Gansu Agricultural University, Lanzhou, Gansu, 730070, P.R.China
}

\begin{abstract}
This paper deals with the progress in the management of reproduction in domestic yaks, including female reproductive biology, male reproductive biology and the main reproductive technologies in both female and male yaks. Further studies and actions with some immediate measures are also recommended.
\end{abstract}

\section{Introduction}

The yak (Poephagus grunniens) belongs to the bovine subfamily Bovidae and is the only species of the genus Poephagus. While the camel is regarded as "a ship of the desert", the yak is reasonably termed as "a ship of plateau" or more precisely, "a sole vehicle of the Asia Highland". The yak is indeed the only large domestic animal which populates the central Asian Highlands up to altitudes of $6000 \mathrm{~m}$. The population of yak worldwide is estimated to be about 14 million, distributed in China, Mongolia, Southern Russia, Tajjikistan, Kirgies, Nepal, Bhutàn, Myanmar, Pakistan and Afghanistan, of which $90 \%$ is located in China. It is therefore of extreme economic importance, providing the indigenous nomads and farmers with the essentials for their livelihood. Milk, dairy products and meat are the main foodstuffs. Skin, wool and dung are utilized to meet requirements for shelter, housing and fuel. The animals are also used in farming and transportation of goods.

Currently, most of the literature on the yak is represented by a wealth of articles in scientific journals, reports and proceedings of technical meetings which are mainly in Chinese or Russian languages. These languages are not generally accessible to international communities, and there are only a few books and papers dealing with the yak written in English, especially about its reproduction. The present paper therefore tries to give an account of the current knowledge, new findings and techniques on all aspects of yak reproduction.

\section{Female reproductive physiology}

Reproductive morphology and follicular systems

The structure of the reproductive organs of the female yak differs in some aspects from those of dairy cows. The cervix in yaks has three transverse circles consisting of many tight folds, with an average length of $5.0 \pm 0.9 \mathrm{~cm}$ and an average external diameter of $3.2 \pm 0.7 \mathrm{~cm}$. The body of the uterus is rather short, being $2.1 \pm 0.8 \mathrm{~cm}$ in length. A long and distinct septum (approx 6 $\mathrm{cm}$ in length) extends downward from the bifurcation of the uterine horns towards the uterine body (Fig. 1). Because of the short uterine body and the long septum, especially the relatively free cervix within the pelvic cavity, the uterus can be readily held for palpation. The length of the uterine tube is $18-24 \mathrm{~cm}$ (Cui \& Yu 1999a). 


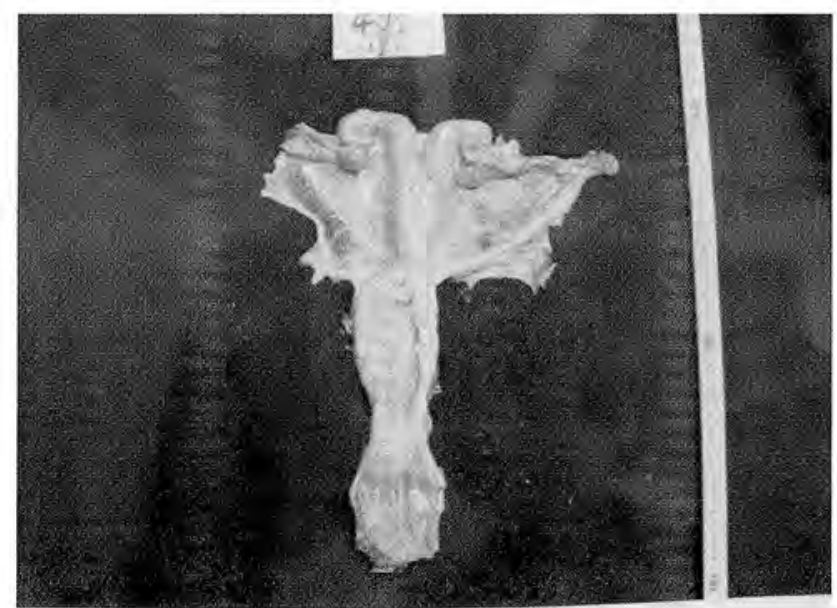

(From one year old yak heifer)

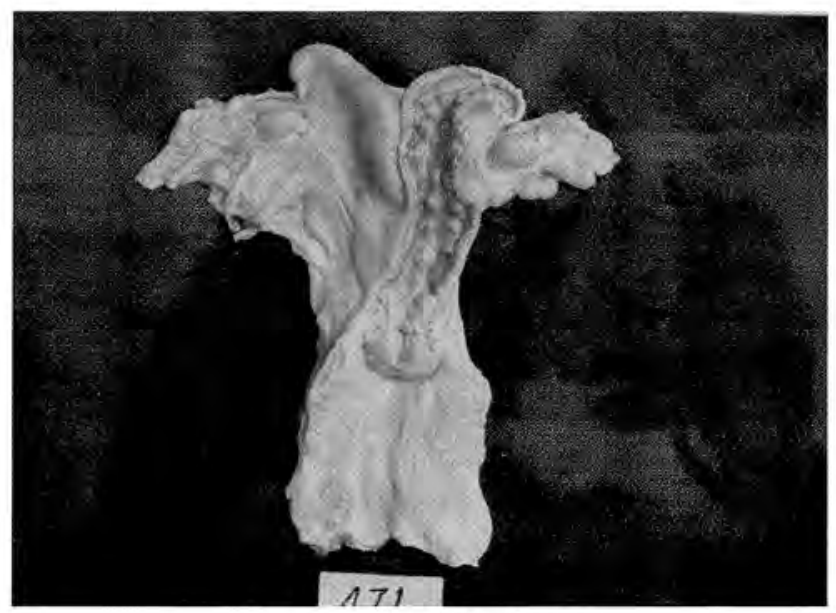

(From 12 year old yak cow)

Fig. 1 The inner reproductive organs of female yaks.

Cui and Yu (1999b) carried out a study on forty-five female yaks of different ages with know reproductive histories to determine the morphology and follicular systems of ovaries. The histological structure of the ovaries resembled that of cattle and buffalo and was similar in animals of different ages. Atresia was recognized at all stages of follicular development: atresia of primordial follicles took one form (i.e, atresia of the oocyte); that of growing follicles could be divided into two stages (early and late); and that of Graafian follicular were classified as early, definite- and late-stage. Details of follicle populations in this study are given in Table 1. 
Table 1. Follicular characteristics of female yaks

\begin{tabular}{lcccc}
\hline Total per oyary pair, & 1-month-old calves & 1-year-old heifers, & 2-year-old heifers & 7-10-year-old cow \\
\hline Primordial follicles (n) & $53,500 \pm 6300$ & $32,870 \pm 4500$ & $22,850 \pm 2800$ & $9500 \pm 1200$ \\
Atretic primordial follicles (\%) & 51.6 & 55.5 & 56.7 & 47.4 \\
Growing follicles (n) & $210 \pm 76$ & $815 \pm 95$ & $895 \pm 142$ & $445 \pm 88$ \\
Atretic growing follicles (n) & $119.5 \pm 21.5$ & $605.5 \pm 74.3$ & $721.6 \pm 78.5$ & $275.8 \pm 66.3$ \\
Graafian follicles (n) & $36.5 \pm 14.2$ & $41.7 \pm 12.3$ & $37.8 \pm 9.8$ & $42.5 \pm 74.5$ \\
Atretic Graafian follicles (\%) & $22.1 \pm 5.6$, & $21.2 \pm 7.6$ & $21.5 \pm 4.7$ & $25.3 \pm 6.7$ \\
\hline
\end{tabular}

\section{Puberty}

First oestrus in yaks generally occurs in the second or third summer and autumn following birth, i.e, at between 13 and 30 months of age. In Mongolia, around 10\% of yaks have their first oestrus in the second summer of their life, but most females do not show estrus until their third summer when they are more than 2 years old. Onset of first oestrus is determined more by the body condition at the beginning of the breeding season than by age (Magash 1991). Very similar results were also reported by Katzina \& Maturova (1989) based on observations on yak in Tuva Autonomous Republic.

Yu \& Li (2001) found, for observation of 60 yak heifers, that cyclic activity began at a mean age of 33 months. In China, the majority of yaks are mated for the first time at the age of 3 years (i.e., in the 4th warm season following birth) but, under favorable conditions, some yaks may be mated a year earlier. Such conditions prevailed among 197 primaparous Jiulong yaks in the Sichuan province studied by Cai (1980). In that study, $32.5 \%$ calved at 3 years, $59.9 \%$ at 4 , $6.1 \%$ at 5 , and the remaining $1.5 \%$ at 6 years of age. At these ages, yaks had reached between $75 \%$ and $100 \%$ of their mature weight. In this context, yaks in Tuva reached fertile estrus at approximately $90 \%$ of mature body weight, compared with $60 \%$ for Bos taurus cattle in that region (Katzina \& Maturova 1989). First mating at an age of 2 years occurs to a limited extent in China but is more common amongst yaks in other countries.

\section{Breeding season}

Yaks are considered to be seasonal breeders. However, information about the breeding season is rather conflicting. The onset and the end of the breeding season are affected by ecological factors such as climate, grass growth, latitude and altitude. When temperature and humidity start to rise and grass starts to grow, the body condition of yaks improves following their long period of deprivation and weight loss over the winter and, at that time, females come into the breeding season. On the northwestern grasslands of Sichuan the season begins around June ( $\mathrm{Hu}$ et al. 1960) whereas at the higher elevation of Laqu in Tibet, the breeding season does not start until July. Similar observations have been reported from Kirgizia, where the annual onset of the breeding season started on May 25th at an elevation of $1400 \mathrm{~m}$ and occurred progressively later until at the altitude of $2700 \mathrm{~m}$ oestrus started after June 22nd (Denisov 1958). The breeding season reaches its peak in July and August when temperature is at its highest and grass growth is at its best. Thereafter, the frequency of oestrus decreases and stops altogether around November. 


\section{Oestrus}

The average length of oestrous cycle in yaks has been reported to be around 18 to 22 days. However, the great variation in the length of the oestrous cycle is one of the problems in yak reproduction. The main reasons for the variation could be silent or non-detected oestrus, delayed ovulation, implantation failure, or embryonic death. Oestrus in yaks is greatly affected by the environment, and when the weather is unfavorable the onset of oestrus is delayed, and when in favorable circumstances the onset of oestrus in female yaks is advanced.

The duration of the oestrus is not easily determined in the yak, since the signs of oestrus are not always clear. Estimates from northwestern Sichun suggest that oestrus lasts 12 to 16 hours. In a study of 41 female yaks (Yu et al. 1993a), oestrus lasted 24 hours or less in 26 of them, whilst 4 yaks showed oestrus for up to 72 hours. More than $80 \%$ of these animals ovulated within $24 \mathrm{~h}$ after the end of oestrus. There is a tendency for the proportion of yaks with heat periods of 1 to 2 days to increase later in the breeding season when ambient temperature begins to decline. Purevzav \& Beshlebnov (1967) recorded that among 54 Mongolian yaks, 26 were recorded in heat for only 0.5 to $6.5 \mathrm{~h}$. A further 17 females showed oestrus for between 6.5 and $12.5 \mathrm{~h}, 7$ females showed oestrus for 12.5 to $18.5 \mathrm{~h}$, and only 4 females showed a longer duration of oestrus. Most of these yaks ovulated 12 to $24 \mathrm{~h}$ after the end of oestrus (Yu et al. 1993a).

Changes in the appearance of the reproductive organs at the time of oestrus are more obvious than behavioral changes ( $\mathrm{Yu}$ et al. 1993a). The vulva becomes swollen and the vagina reddens. Mucus is discharged from the vulva in a majority of oestrous females, although a substantial minority shows no such discharge. The vagina and cervix dilate, the female tends to raise her tail and urinates frequently. As in other cattle, yak females on heat search out and ride other females and like to be approached by male yaks. However, these signs are less pronounced than in Bos taurus cattle. The most pronounced signs of oestrus are being followed and mounted by mature bulls, swelling of the vulva, reddening of the vaginal mucosa and the discharge of mucous.

The average duration of post-partum anoestrous at Xiandong farm in Sichuan Province was reported to be 125 days (Cai \& Weiner 1995); that figure, however, was subject to much variation. However, the duration of the postpartum aneostrous period was much shorter $(70.5 \pm 18.5$ days) for yaks in good body condition than for those in poor body condition $(122.3 \pm 11.8$ days; Liu \& Liu 1982). The duration of postpartum anoestrous period has been reported to be related to month of calving: for females calving in March, April, May and June, the periods were 131, 124, 90 and 75 days respectively, whilst for animals calving between March and August, the periods for each month were 134, 130, 105, 89 and 37 days (Lei 1964).

Likewise, Magasch (1990) provided results on the interval between calving and first postpartum oestrus for yak females in Mongolia, showing clearly a relationship with month of calving, the earlier the calving the longer the interval. However, there was considerable variation in these results around the average interval periods. Magasch (1990) reported that the service period following calving showed a very similar seasonal pattern for the interval between calving and first postpartum oestrus.

\section{Gestation and parturition}

Conception rates after mating at the first oestrus of the breeding season are generally high. In a trial with 265 yaks that had previously calved, $72.4 \%$ became pregnant following the first oestrus, a further $23.4 \%$ following the second, and $3.4 \%$ and $0.8 \%$ following the third and fourth cycles respectively (Liu 1981). In a similar investigation of 342 yaks in Mongolia, it was found that $70.5 \%$ were pregnant after the first service, $19.3 \%$ conceived to a second service 
and $4.6 \%$ to a third service; giving an overall pregnancy rate of $94.4 \%$ (Magasch 1990). It also appears from the above results that Mongolian yaks that fail to conceive at their first oestrus are capable of returning to oestrus up to three times in the same breeding season. In one particularly well-maintained herd of yaks on grassland in Gansu, in which animals received some supplementary feed in late winter and early spring, a conception rate of $93.4 \%$ was achieved (Yu et al. 1993a).

Almost all births take place during the day and only very few at night when yak cows are normally at the herders' campsite. Typical behavior of the yak during labour includes lying on her side and standing up again for delivery. Dystocia is a rare occurrence in yak carrying purebreed calves. The umbilical cord is severed by mechanical stretching as the cow gets up or as the calf falls down after delivery. Yak cows with hybrid calves, however, require help for delivery. For example, there were 28 cases of dystocia among 681 such calvings $(4.1 \%)$ over a period of 10 years in one study in Sichuan (Xu 1964). Twins are rare and represent only about $0.5 \%$ of all births, but higher rates have exceptionally been recorded (Cai \& Weiner 1995). The dam generally licks the newborn calf for about $10 \mathrm{~min}$, after which the calf attempts to stand up and suckle.

The placenta is normally passed within half an hour of parturition, although this may not occur until up to 6 hours later. The dam is intensely protective of her calf in the period shortly after birth and will attack any person coming close. Bonding between dam and calf depends mainly on smelling and licking. Longer parturition times and dystocia militate against such bonding and thus place Pian Niu (hybrid) calves at a disadvantage versus purebred yak calves.

Yu \& Chen (1994a) studied a total of 1953 yak cows ( 3 to 11 years of age) in two populations differing in calving rates. The calving rate was $78 \%$ in the high calving rate group and $50 \%$ in the other. It appears that the main differences were due to milking frequency and feed supplementation between the two populations. The high calving rate group was milked once daily and received supplementary feed from late winter to early spring, whilst the low calving rate group was milked twice daily and did not received any supplementary feed.

Calving interval is an important economic index in the yak industry. Wang et al. (1997) observed 439 calvings from 161 yaks over a 7 -year period. The average calving interval was $459 \pm 131$ days and showed a tendency for gradual decrease with increase in parity.

\section{Reproductive endocrinology}

Since 1985 we have carried out a series of studies to examine the hormonal control of puberty, the oestrous cycle, pregnancy, parturition and the postpartum period in the yak ( $\mathrm{Yu}$ et al. 1993b; 1993c; Yu \& Chen 1993; 1994b; 2000; Yu \& Li 2001).

Fifteen yak heifers were used to analyze plasma progesterone before and at the attainment of puberty. They were divided into three age groups: group $1(10 \sim 14$ months, $n=5$ ), group 2 $(20 \sim 24$ months, $n=5)$ and group $3(30 \sim 36$ months, $n=5)$. Group 1 yak heifers were found to have two progesterone profiles: 10 (inactive ovary) profile and LPSC (low progesterone short cycle) profile; group 2 had three profiles: 10 profile, LPSC profile and LPNC (low progesterone normal cycle) profile; group 3 had three profiles: LPSC profile, LPNC profile and a normal estrous cycle profile. It would be concluded that one or two, or even more brief rise(s) in circulating progesterone were present in yak heifers. These rises, however, were not followed by a normal luteal phase except in two yak heifers that came into oestrus.

In yak cows, the LH surge appeared $12-15 \mathrm{~h}$ after the onset of oestrus. Concentrations of oestradiol-17ß were high $(28.07 \pm 7.24 \mathrm{pg} / \mathrm{mL}$ in plasma) at the onset of oestrus, but continued to increase thereafter, reaching peak values $(49.88 \pm 10.58 \mathrm{pg} / \mathrm{mL}$ in plasma) about $2 \mathrm{~h}$ after the 
$\mathrm{LH}$ surge; then it fell. Progesterone concentrations were low at the onset of oestrus, rose markedly within $16 \mathrm{~h}$ and reached a small peak $18 \mathrm{~h}$ after the onset of oestrus (i.e. about $4 \mathrm{~h}$ after the LH surge). However, concentrations promptly dropped thereafter, with similar values at $20 \mathrm{~h}$ as had been present at the onset of oestrus (Fig. 2).
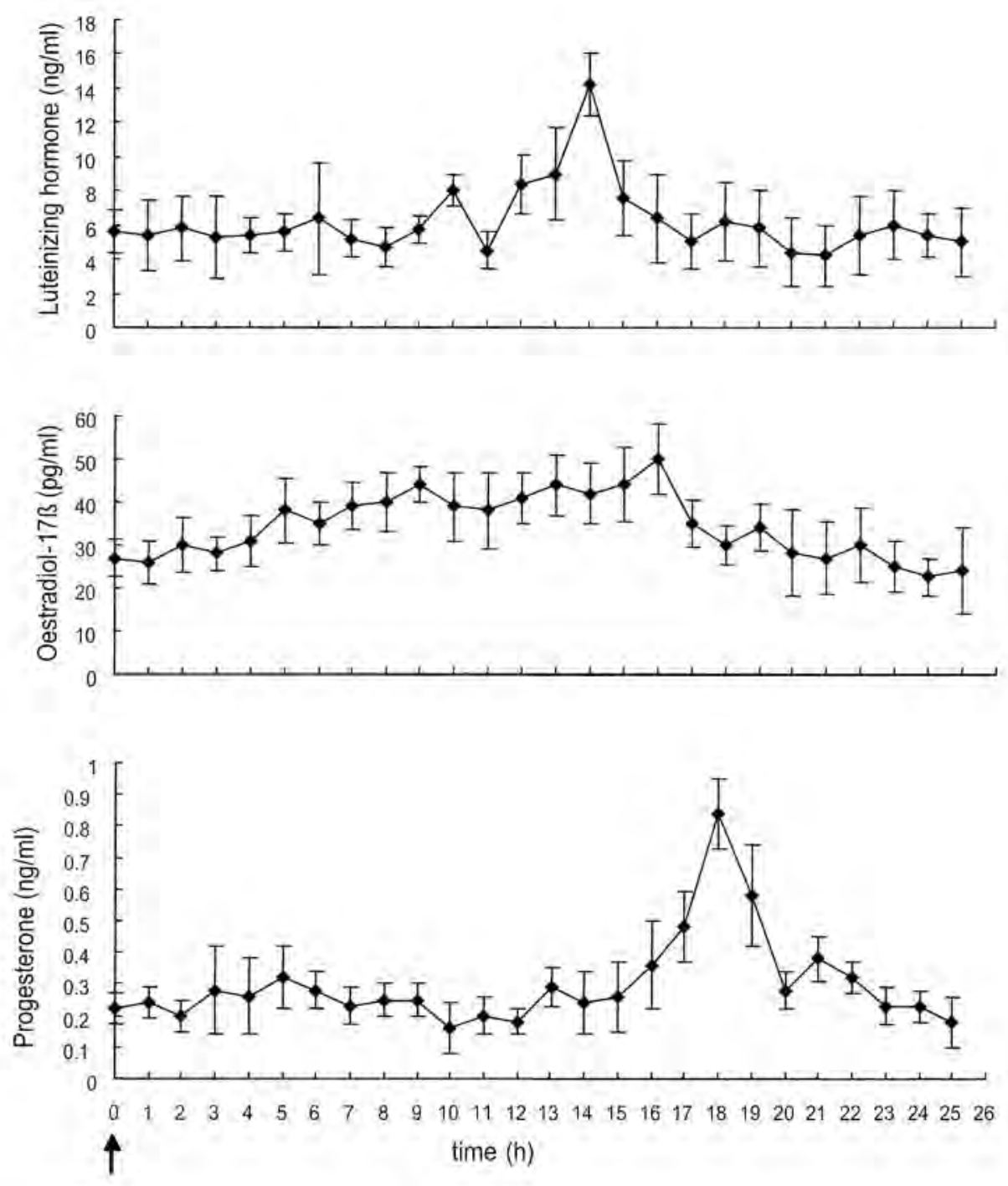

Oestrus

Fig. 2 Mean concentration ( $+S D)$ of luteininizing hormone $(n=6)$, oestradiol-17B $(n=5)$ and progesterone $(n=5)$ in peripheral plamsa taken at $1 \mathrm{hr}$ intervals around the time of oestrus.

Concentrations of oestradiol- $17 \beta$ and progesterone in plasma and milk of the six yaks during normal cycles were measured. There were three peaks of oestradiol-17ß in plasma and milk on the day of oestrus, and on days 5 and 14 of the cycle. Progesterone levels in plasma and milk 
were low during oestrus but peaks were seen on day 15 . The levels of oestradiol-17ß and progesterone in milk were about 4 or 5 times higher than those in plasma during the normal cycle. Three yak cows were used to determine the oestradiol-17ß and progesterone profiles in plasma during the short cycle (Fig. 3). It was found that the pattern of both oestradiol-17ß and progesterone during the short cycle were similar to those during the normal cycle, but the values were lower. The result indicated that some follicles must have been developing; this was confirmed in all 3 cows examined rectally. We also found that all yak cows showed some oestrus-like symptoms and some of them presented a short cycle at the beginning of the breeding season before a normal cycle. It might be concluded that a transition to the normal cyclic activity occurs at the beginning of the breeding season in all yak cows.
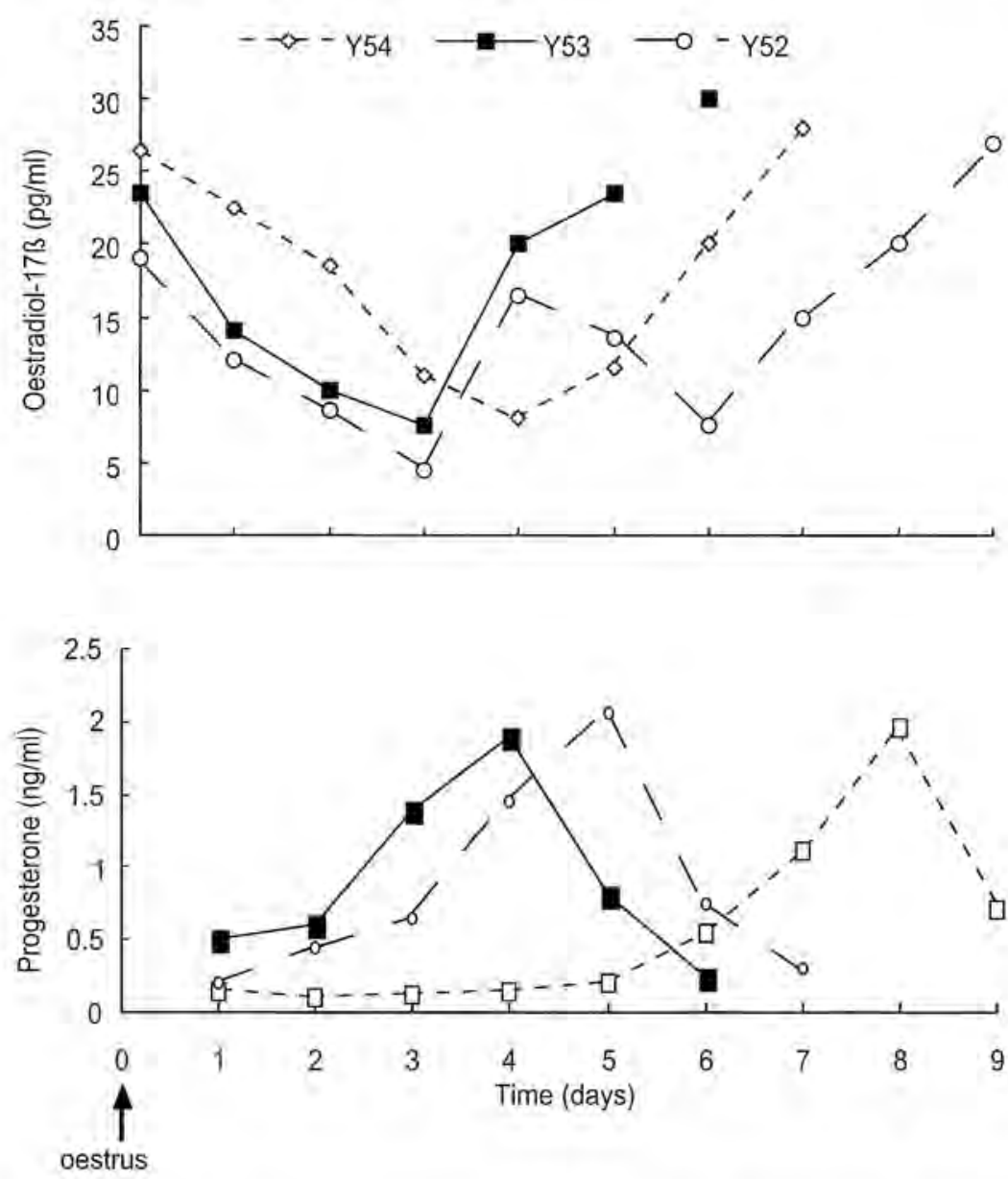

Fig. 3 Concentrations of plasma progesterone and oestradiol-17ß during the short oestrous cycle in three yaks.

The profiles of progesterone were similar in pregnant and non-pregnant yaks over the first 14 days following oestrus, but concentrations were significantly higher in pregnant than non-pregnant yaks on Day 19, and tended to progressively increase thereafter. Plasma progesterone concentrations declined around Day 120 , then increased to reach its maximum on Day 210; declined again 20 days before parturition and were basal by the time of parturition. Oestradiol- 
$17 ß$ concentrations in plasma and milk increased gradually from Day 23 post-conception, decreased abruptly on Day 60 , then increased to reach maximum values at parturition. After parturition, oestradiol concentrations returned to baseline.

After calving, four patterns of milk progesterone profiles in suckled yak cows were noted. Type I: Cows had cyclic changes of milk progesterone concentrations within 40 days postpartum. Type II: Cows had a short progesterone rise within 20 days postpartum, then progesterone concentrations remained low $(<0.5 \mathrm{ng} / \mathrm{ml})$ until 90 days postpartum. Type III: Cows had milk progesterone concentrations of below $0.5 \mathrm{ng} / \mathrm{ml}$ until 90 days postpartum. Type IV: Cows had milk progesterone concentrations of above $1.0 \mathrm{ng} / \mathrm{ml}$ from 20 days until 90 days postpartum.

\section{Male reproductive physiology}

\section{Testis and sperm morphology}

The yak scrotum is small, with abundant hair, which is apparently an adaptation to the cold environment (Xu 1964). Yak testes are similar anatomically and histologically to those of other bovine bulls, except that they are smaller that those of B. taurus bulls (approximately 300 versus $550-650 \mathrm{~g}$ ). In general, the testicular weight in Charolais and Holstein bulls is $\sim 2 \mathrm{x}$ that of yak, while that of Zebu was $\sim 0.65 \times$ (Yan et al. 1997). The height of the seminiferous epithelium and volume density of seminiferous tubules and seminiferous epithelium increase with age; but the volume density of the lumen of the seminiferous tubule and interstitial tissue decrease gradually with age. At 24 months of age, the volume density of seminiferous tubule of yak is $0.786 \mu \mathrm{m}^{3} / \mu \mathrm{m}^{3}$, the volume density of seminiferous epithelium is $0.677 \mu \mathrm{m}^{3} / \mu \mathrm{m}^{3}$, the height of seminiferous epithelium is $85.66 \mu \mathrm{m}$ and the volume percentage of seminiferous tubules is $78.6 \%$. The height of seminiferous epithelium and the capacity rate of seminiferous tubule in yak bulls at 24 months of age is $85.66 \mu \mathrm{m}$ and $78.58 \%$, respectively. These figures are similar to those in mature males of common cattle breeds (79.4\%; Yan et al. 1997).

\section{Spermatogenesis}

The yak sperm consists of a head, neck and tail that are very similar to those of other cattle breeds. Compared to sperm from B. taurus bulls, the dimensions for yak sperm are 9.50 versus $8.32 \mu \mathrm{m}$, the length of the midpiece is 14.20 versus $14.40 \mu \mathrm{m}$, and the length of the principal piece is 51.83 versus. $47.5 \mu \mathrm{m}$ (Xia et al. 1990a).

Spermatogenesis in the yak is similar to that in other species inasmuch as it is divided into three major processes: spermatocytogenesis, meiosis and spermiogenesis. Xia et al. (1990c) reported that the spermatogenetic cycle and the seminiferous epithelium phase of yak bulls are very similar to that of cattle. It appears that Phases VII and XI are very long, whilst Phases IX, X, and XII are rather shorter than in cattle.

Xia et al. (1990b) concluded that the numbers of both Sertoli cells and all kinds of germ cells in yak testes are approximately $80 \%$ of those in cattle: a difference which was attributed to both intrinsic differences between the species and the effects of nutrition. The rate of seminiferous cell production in yaks is similar to that in cattle during spermatogenesis; however, the proliferation rate in yak is about $10 \%$ lower than in cattle. Hence, daily sperm production in yak is maintained at a point somewhere between that of $B$. taurus and $B$. indicus cattle

\section{Sexual activity}

Males start to show mounting behaviour around 6 months of age. This behaviour continues and 
intensifies to include searching and mounting females in the following year. No sperm, however, are found in epididymal fluid of yak bulls before the age of 2 years. Puberty thus occurs in the third warm season following birth, when the males are over 2 years old. In practice, bulls start to mate from the age of 3 , reaching their peak ability at around 6 to 7 years old after establishing their dominant position in the mating hierarchy of the herd by fighting. After 8 years of age, yak bulls start to lose to younger bulls in the competition for females (Zhang 1994).

A sexually productive life expectancy of not more than 10 years for the yak bull was suggested by results from an artificial insemination (Al) stud farm of 38 yak bulls in Tibet. The ejaculate volume, concentration and motility of sperm rose steadily from the age of 3 to 9 years old and then declined. Semen quality and quantity also change with season. In Dangxi, Tibet, the highest values for volume, sperm concentration and motility were obtained from June to September, with the peak occurring in September (Zhang 1994).

In Mongolian yaks, the older the bull the more females it is able to serve; which is consistent with the courtship behavior and dominance hierarchy of bulls. However, it was also found that the younger bulls, with fewer females at their disposal, mounted their mates more frequently (Magash 1991). Fertilization seemed to be more dependent on the number of services than on the age of the bull, which shows that overall pregnancy rate of females increased with the number of services.

\section{Reproductive biotechnologies}

\section{Pregnancy confirmation}

Various methods for the determination of pregnancy have been developed in the yak, but only method which has been investigated sufficiently to be recommended for use in practice is the clinical method of rectal palpation. This method is the same as for cattle and buffalo. Early pregnancy diagnosis can be made (Yu et al. 1993a) by examining the changes in the uterus (the uterine horns are of equal size, but became flaccid), the furrow between the bases of the horns (become less distinct) and especially the size of the ovaries (the ovary on the gravid horn side is significantly enlarged to about twice the size of the other one).

Assaying for progesterone is another method for pregnancy diagnosis, based on the presence of continuously elevated progesterone concentrations during pregnancy. Blood should be sampled $20 \sim 24$ days after mating and progesterone be assayed by radioimmunoassay. Females with blood progesterone concentrations above $1.0 \mathrm{ng} / \mathrm{ml}$ are considered to be pregnant (Yu et al. 1993b).

\section{Oestrus synchronization}

Oestrus synchronization in the yak has been effectively accomplished with products commonly used in cattle, including $\mathrm{PGF}_{2 \alpha}$ and progesterone. Synchronization of oestrus is most effective during the breeding season, but it is also possible to induce oestrus outside the breeding season. Regardless of the method used, considerable effort must be made to minimize stress, or fertility will be adversely affected.

When $\mathrm{PGF}_{20}$ is used, the animal must be cycling and have a responsive corpus luteum. Following a single injection of $\mathrm{PGF}_{2 \alpha}$ (during the ovulatory season), approximately $50 \%$ of yaks will be detected in oestrus, but the interval from treatment to the onset of oestrus is usually slightly longer than in cattle. A second injection of $\mathrm{PGF}_{2 \alpha} 11$ days after the first will effectively 
synchronize the group. In one study (Magash 1997), 135 yaks were administered twice daily in $2.0 \mathrm{ml}$ and $5.0 \mathrm{ml}$ doses with two $\mathrm{PGF}_{2 \alpha}$ preparations (Oestrophan \& Enzaprost; the former was made in Czech and later in Hungary). Most animals ( 55 to $70 \%$ ) were detected in oestrus 3 days after treatment. However, there was a seasonal effect on the proportion in oestrus, with $50.0 \%$ and $46.2 \%$ detected in oestrus following administration of the two products, respectively, in July and a better response rate $(61.5 \%$ and $58.3 \%)$ in August. Following two treatments at 10-day intervals, the oestrus synchronization rates were $86.2 \%$ and $90.0 \%$, and the conception rates following insemination with frozen-thawed Holstein semen were 77.9 and $78.9 \%$ for the two products respectively.

\section{Inducing oestrus}

Yu et al. (1996) used various exogenous hormones (LRH-A3, FSH, eCG and hCG) to induce oestrus in 180 female yaks of different ages, The animals included 80 milking yak-cows that had calved and had their calves with them throughout the experiment year, 80 non-milking cows that had not calved during the experiment year, and 20 heifers. After a single intramuscular injection of $\mathrm{LRH}-\mathrm{A} 3$, the non-milking yak-cows showed the best response, with an oestrus rate of $95 \%$. Yak heifers responded with an oestrus rate of $80 \%$, which was better than occurred in the milking yak cows. The oestrus rates were $82 \%$ and $88 \%$ respectively in the non-milking yak-cows after eCG or hCG injection. The oestrus rate in the milking yak-cows reached $70 \%$ only after a combined treatment of an intramuscular injection of $\mathrm{FSH}$ on day 0 and $\mathrm{LRH}-\mathrm{A} 3$ on day 2 . Oestrus in treated yaks occurred within 1 to 10 days after normal treatment, with the non-milking yak-cows and heifers showing oestrus earlier than milking cows. While there is considerable variation in fertility after oestrus induction in female yaks in this study, overall pregnancy rates were $86 \%$ in non-milking yak-cows and $73 \%$ in milking yak-cows.

\section{Superovulation}

Superovulation has been attempted in yak females using a variety of treatments and under varying conditions. However, satisfactory responses have not been consistently achieved. In a small trial (Zagdsuren et al. 1997), 3 barren females ( $4-8$ years old) were given an intravaginal insert containing $1.2 \mathrm{~g}$ progesterone (CIDR) for 12 days, injected with $\mathrm{PGF}_{2 \alpha}$ (Lutalyse) on the third day after the first injection of FSH and inseminated $24-36$ hours after the last injection of FSH. The average number of corpora lutea and non-ovulated follicles were $5.0 \pm 0.6$ and $1.3 \pm 0.9$, respectively, but embryo recovery was not reported.

Davaa et al. (2000) used FSH and PMSG to induce superovulation in yak cows. Oestrus was detected $34.1 \pm 0.52 \mathrm{~h}$ after the prostaglandin treatment. The average number of ovarian follicles was $5.4 \pm 0.65$ and $4.5 \pm 0.43$, of which $2.6 \pm 0.30$ and $1.9 \pm 0.20$ ovulated from the right and left ovaries, respectively.

\section{In vitro embryo production (IVP)}

Despite success in other species, there are no reports of yak calves being produced following in vitro maturation and fertilization of oocytes collected from nonstimulated females. However, it is noteworthy that these embryos are capable of developing to the blastula phase in vitro.

Using standard procedures, Chen et al. (1997) studied the efficiency of IVP methods in yak and local breeds of cattle. Results showed that the cleavage and blastocyst rates for yak IVF 
were $35 / 56(62.5 \%)$ and $9 / 56(16.1 \%)$, respectively, indicating that these procedures, derived from those used in cattle, produced acceptable results which were at least as good as those obtained with IVP using oocytes and semen from local cattle.

Luo \& Yang (1994) obtained ovaries from 4 yaks after slaughter. In this study, 25 oocytes were aspirated from 8 ovaries and, after incubation for 20 h, 20 oocytes developed to meiosis II. Fertilization and cleavage rates were $12 / 20(60 \%)$ and $8 / 20(40 \%)$, respectively. Although two normal morrulae were transferred into the uterus of a Holstein-Friesian cow, she was subsequently diagnosed nonpregnant.

In our laboratory, He \& Cui (2005) carried out a comparative study to improve the techniques of IVP in yak. They established a procedure that achieved a cleavage rate of $62.2 \%$, and the developmental rates of 4 -cell and 8 -cell were $46.7 \%$ and $28.9 \%$, respectively.

\section{Embryo transfer}

Although further studies are still needed to optimize the procedures of superovulation, oestrus synchronization, embryo recovery and transfer in yaks, we have made a successful embryo transfer in Tianzhu white yaks (Ju \& Yu 2006). In our experiment, a comparative test was taken and a standard protocol was determined as follows. The donors (Tianzhu white yak) received a CIDR-B program containing $1.9 \mathrm{~g}$ progesterone at an undetermined stage of the oestrous cycle (Day 0). Superovulation was induced with $10 \mathrm{mg}$ of p-FSH, divided into eight decreasing doses at intervals of $12 \mathrm{~h}$ from Day 5 . The donors once again received two injections of $\mathrm{PGF}_{2 \alpha}$ on Days 7 and 8 and CIDRs were removed in the afternoon of Day 8 . Al was performed 12 and $22 \mathrm{~h}$ after the onset of behavioural oestrus and embryos were collected 7 days after Al. The mean numbers of total corpora lutea, non-ovulated follicles, viable (transferable) and degenerated embryos were $4.8 \pm 2.2,1.1 \pm 0.8,2.5 \pm 1.3$ and $1.4 \pm 0.9$, respectively. Twelve viable embryos were finally transplanted to 10 oestrus-synchronized black yak females (recipients). As a result, 6 of the 12 black yak females became pregnant. All 6 animals delivered full-term calves normally. The results of our study are the first confirmation that the embryo transfer is feasible in the yak.

\section{Interspecies hybridization and Al}

Hybridization between yak and cattle of other species is recorded in ancient historical records. The cattle originally used were local breeds, generally referred to as 'yellow' cattle (B. taurus) in China, and both B. taurus and B. indicus (Zebu) cattle elsewhere. Although this practice continues, more recently improved breeds of cattle have also been used (facilitated by $\mathrm{Al}$ of frozen-thawed semen). However, due to the reproductive isolation of different animals and the low conception rate of hybrids produced from yak and other cattle breeds, crossbreeding between yaks and cattle is limited to only the F1 and F2 generations. The F3 generation (containing about $12.5 \%$ of yak genetics) have difficulty surviving in the high-altitude areas (above $3000 \mathrm{~m}$ ) in the Qinghai-Tibet Plateau and therefore are seldom produced. Since Al is inevitably restricted to more accessible areas and maintenance of improved breeds is difficult and expensive, most crossbreeding programs use locally available cattle.

Although crossbreeding and the infertility of male hybrids have been extensively studied, there have been no clear conclusions. The ability of hybrids to produce sperm increases gradually with successive generations. Crossbreeding is generally restricted to the F1 and F2 generations, but sperm production does not resume until at least the third backcross (15/16 yak or cattle), and often not until the fourth backcross. 
Semen from improved breeds of cattle, such as Holstein-Friesian and Simmental, have been used to breed yaks but are not popular due to the lower survival of the hybrids. Recently, semen from Hereford, Angus, Simmental, Limousin and Charolais cattle has been used extensively to breed yak females. In Nepal, Zebu cattle are regularly crossed with yaks. It has been suggested that crossing yaks with Highland cattle (from the United Kingdom) results in hybrids with superior productivity.

Experiments were conducted in the 1920's and 1930's (at Buffalo Park, Wainwright, Canada) to develop a meat animal for their cold northern conditions. In these experiments, a small number of crosses were successfully made between yak males and female American bison and half-bison (bison $x$ cattle cross) (Deakin et al. 1935).

\section{Conclusions and recommendations}

Although it is a credit to those involved that much scientific investigation is done with the reproduction of yak, in spite of the difficulties, it is also important to acknowledge that large gaps in knowledge still exist. Therefore, the following general policy guidelines are recommended for the further studies:

Reproductive performance: yaks under the existing management conditions breed once in two years though some of the yaks are annual breeder. Delayed sexual maturity, the long postpartum period, and seasonality of breeding are some of the reproductive problems confronted in the yak husbandry. The age of sexual maturity has been markedly reduced in the other domestic cattle by selective breeding. Since it is clear that, besides genetic make up, inadequate nutrition including deficiency and imbalance of minerals contribute to delayed maturity, there are a number of possible strategies to use to achieve a reduction in the age of sexual maturity. Indeed, it is likely that once the nutrition is addressed properly, many of the existing problems of reproductive performance in yak would be automatically overcome. The long winter, with scanty feeding resources, keep yaks in a maximum of physiological stress which also impacts on other systems.

Reproductive physiology: Basic information of embryology in yak is scanty. Information on factors including hormones and other related materials which may play a major role in various reproductive periods is still limited. However, studies in this area should mainly be concentrated on physiology of reproductive adaptability to the extreme environment.

Breeding: Crossbreeding of yaks should only be encouraged up to F1 and should preferably utilize a breed of bull with high production potential but smaller in size to avoid parturition complications. The F1 male will be made available to the different pockets on exchange with the non-description in bred bulls. Meanwhile, breeding should be restricted within the breeds/ types to conserve and preserve genetic identity of each breed.

Reproduction and nutrition: There is a paucity of information on whether natural yak diets have deficiencies, excesses or imbalances of, for example, trace minerals. So more detailed study about the relationship of yak reproductive physiology and nutrition are needed to determine whether specific nutrients may be limiting factors and whether specific supplements at critical times would be cost-effective.

Reproductive technologies: Although the study of reproductive events in yak has generally 
lagged far behind that of domestic livestock, recent interest and investigations have in part corrected this deficit. Nonetheless, there is still much to be learned before these technologies are commonplace in yak. In that regard, short-term preservation of yak ovaries and/or oocytes, and cryopreservation of oocytes and embryos would be of considerable benefit. Remarkably, many techniques developed and proven in cattle have been adapted to yak with only a few, minor, modifications. The success of IVP and embryo transfer techniques in yak suggests that there are many opportunities for similar approaches in other animals. In that regard, IVM and IVF utilizing epididymal spermatozoa and IVC with oviduct cell co-culture are likely to be of use in many different species in the near future. It is expected that this will promote assisted reproduction in exotic and endangered species and facilitate opportunities for the international movement of gametes.

Overall, it is now widely recognized that many of the questions at a fundamental and a practical level, which it is hoped would lead to an improvement in yak productivity, are concerned not with single issues, or single characteristics of the yak, but with the understanding of complex interactions. For example, it may no longer be necessary to ask what hormones are involved in affecting reproductive rate, but how hormonal responses to nutritional deprivation affect calving intervals and how specific nutrient inputs at critical times may improve matters. It is possibly no longer necessary to ask about the occurrence of heterosis from interspecific hybrids with yak, even though the actual levels of heterosis are still largely unknown, but it is more relevant to current practice to ask how such heterosis is expressed at different levels of nutritional and environmental stress and how it fits into a comprehensive breeding strategy.

Cooperative and coordinated design, execution and analysis of experiments and development trials with yak may well provide a total benefit which is greater than the sum of its parts. It deserves serious further consideration.

\section{Acknowledgements}

Grateful acknowledgments to International Foundation for Science (B/1424-1\&2; B/1696-1\&2), National Natural Science Foundation of China (39100096, 39970546 \& 30371069), Gansu Science Foundation of Application of Biological Techniques in Agriculture (GNSW-2004-07) and Science Foundation of Gansu Province (2GS042-A41-001-05) for funding our studies.

\section{References}

Cai L 1980 Studies on the reproductive organs of female yak Journal of Chinese Yak 3 10-16.

Cai L \& Winer G 1995 The Yak. Regional Office for Asia and the Pacific of the Food and Agriculture Organization of the United Nations, Bangkok, Thailand.

Chen JB, Hai LQ \& Yi H 1997 Comparison on effect on in vitro fertilization between the yak and ordinary cattle. Proceedings of the Second International Congress on Yak China 195-197.

Cui Y \& Yu SJ 1999a An Anatomical study of the internal genital organs of the yak at different age. The Veterinary lournal 157 192-196.

Cui Y \& Yu SJ 1999b Ovarian morphology and follicular systems in yaks of different ages. The Veterinary Journal 157 197-205.
Davaa M, Altankhuag N \& Zagdsuren Y 2000 The preliminary experiment to induce superovulation in female yaks. International Yak Newsletter 578 (Abstract).

Deakin A, Muir GW \& Smith AG 1935 Hybridization of domestic cattle, bison and yak. Report of Warinwright experiment. Publication 479, Technical Bulletin 2, Dominion of Canada, Department of Agriculture, Ottawa.

Denisov VF 1958 Domestic yak and their hybrids. Selhozgiz P116.

He JF \& Cui Y 2005 Effect of fertilization medium and time on in vitro fertilization of follicular oocytes of yaks. Chinese Journal of Veterinary Science and Technology 11 900-903. 
Hu AG., Cai L \& Du SD 1960 An investigation on yak in Ganzi County. Journal of Southwest Nationality College 4 46-50.

Ju XH \& Yu SJ 2006 Successful embryo transfer in Tianzhu white yaks by standard protocol. Animal Reproduction Science (In Press)

Katzina EV \& Maturova ET 1989 The reproductive function of yak cows. Animal Breeding Abstracts 35258.

Lei HZ 1964 Observation on the genital, physiological and reproductive features of yak, Journal of Chinese Animal Science 7 1-3.

Liu WL \& Liu SY 1982 The observation and analysis for female yaks' characters of Qinghai yak. loumal of Chinese Yak 4 5-7.

Liu ZQ 1981 The reproductive characters of Qinghai yak. Journal of Chinese Yak 4 5-7.

Luo XL \& Yang YZ 1994 Research on in vitro maturation and development of oocyte in slaughtered yak. Proceedings of the First International Congress on Yak China 311-313.

Magasch A 1990 Statische Massmalen diagnostischer Zuchthygienemerkmale bei Yakkuhen in der Mongolei. Wiss Zeitschrift der Humboldt Universität zu Berlin R. Agrarwiss. 39 359-366.

Magash A 1991 Ergebnisse von Untersuchungen uber die Physiologie des Sexualzyklus beim weiblichen Yak. Monatschefte fur Veterinärmedicin 46 257-258.

Magash A 1997 The use of biotechniques in yak reproduction. Proceedings of the Second International Congress on Yak China 175-178.

Purevzav Z \& Beshlebnov AV 1967 Some data on the physiology of reproduction in the yak. Zhivotnovdstvo Mask 29 92-94.

Wang MQ, Li PL \& Bo JL 1997 Yak calving interval and calving efficiency. Proceedings of the Second International Congress on Yak China 29-31.

Xia LJ, Ben ZhK \& Liu H 1990a Ultrastructure of yak spermatozoa. Journal of Chinese Yak 3 4-7.

Xia LJ, Liu H \& Lai MR 1990 b Analysis on the spermatogenesis in yaks and comparison with common cattle. Journal of Chinese Yak 3 12-17.

Xia LJ, Liu H \& Tian HP 1990 c Seminiferous cycles in yak bulls. Journal of Chinese Yak 3 8-11.

Xu KZ 1964 The study on the histology and anatomy of reproductive organs and the reproductive functions of yak and its hybrid. Journal of Chinese Animal Science 3 18-21.
Yan P, Liu ZL, Pan HP \& Zi DJ 1997 Quantitative histology studies on the testes in hybrid bull of wild and domestic yak. Proceedings of the Second International Congress on Yak China 167-169.

Yu SJ \& Chen BX 1993 Traditional systems of management of yak cows in China and observations on reproductive characteristics using milk and plasma progesterone. Strengthening Animal Reproduction Research in Asia through the Application of Immunoassay Techniques Vienna 127-135.

Yu SJ, Huang YM \& Chen BX 1993a Reproductive patterns of the yak, 1. Reproductive phenomena of female yak. British Veterinary lournal 149 579-583.

Yu SJ, Huang YM \& Chen BX 1993b Reproductive patterns of the yak. If. Progesterone and oestradiol$17 B$ levels in plasma and milk just before the breeding season; also during normal and short oestrous cycles. British Veterinary Journal 149 583-593.

Yu S], Huang YM \&Chen BX 1993c Reproductive patterns of the yak. III. Levels of progesterone and oestradiol-1 $7 ß$ during pregnancy and the periparturient period. British Veterinary Journal 149 595-601.

Yu SJ \& Chen BX 1994a Traditional systems of management of yak cows in China and observations on reproductive characteristics using milk and plasma progesterone. Proceedings of the Final Research Coordination Meeting of an FAOIIAEA Coordinated Research Programmer Thailand 127-136.

Yu SJ \& Chen BX 1994b Postpartum ovarian function in yak cows as revealed by concentrations of progesterone in defatted milk. Recent Advances in Animal Production 393.

Yu SJ \& Chen BX 2000 Peripheral concentrations of luteinizing hormone, oestradiol- $17 B$ and progesterone around estrus in six yaks. The Veterinary Journal $\mathbf{1 6 0} \quad 157-161$

Yu SJ \& Li FD 2001 Profiles of plasma progesterone before and at the onset of puberty in yak heifers. Animal Reproduction Science 65 67-73

Yu SJ, Liu ZP \& Chen BX 1996 Use of exogenous hormones to induce oestrus in yak. International Yak Newsletter 2 31-34.

Zagdsuren Yo, Davaa M, Magash A \& Altankhung N 1997 Superovulation in remale yaks. Proceedings of the Second International Congress on Yak China 193-194.

Zhang Y 1994 The relationship between season and age of stud yak bull in Dangxin. Proceedings of the First International Congress on Yak China 303-307. 